\section{Direct computation of instability points with inequality constraints using the FEM}

\author{
H. Tschöpe and P. Wriggers \\ Institut für Baumechanik und Numerische Mechanik, \\ University of Hannover, Hannover, Germany \\ E. Oñate \\ E.T.S. Ingenieros de Caminos, Canales y Puertos, \\ Universidad Politécnica de Cataluña, Barcelona, Spain
}

Keywords Structures, Instability, Constraint handling, Finite element method, Inequality

Abstract The subject of this paper is the computation of instability points in mechanical problems with the finite element method. The objective is to extend the application of critical point detection methods to problems with inequality constraints originating from damage and contact. A simple bilinear model is considered for the damage problems. A bilateral, frictionless contact formulation is used for the contact problems. Among the critical point detection methods the focus is laid on the critical displacement method and the extended system. At first a possible combination of both methods is evaluated by applying them to damage problems. A prediction method based on the extended system is developed to facilitate the comparison of both methods. Secondly, the extended system is used as a computation method for critical points in two-dimensional contact problems.

\section{Introduction}

The history of stability theory for mechanical problems dates back to the 18th century and the early works of Euler (1774), who studied the buckling of beams. A lot of experimental and theoretical investigation has been dedicated to the buckling of plates and shells at the beginning of the 20th century. When numerical methods such as the finite element methods (FEM) were developed, more complex mechanical problems could be solved. With the introduction of arclength methods by Riks (1972) and Wemper (1971) in engineering, even the computation of complex non-linear load-deflection paths became feasible. Detection of critical points, however, was made with indicator functions that had to be computed accompanyingly to incremental-iterative path computation methods. Examples of these are the determinant of the tangent stiffness matrix or the number of negative diagonal elements.

With the extension of the set of equations by a condition for critical points the direct computation of a critical point became feasible. In the mathematical literature these methods can be found in the work of Abbott (1978) and
Direct computation of instability points

611

Received May 2002 Revised December 2002 Accepted January 2003 
EC

$20,5 / 6$

\section{2}

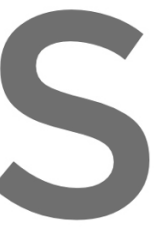

Seydel (1979). The extended system was applied to engineering problems for the first time by Wriggers et al. (1988). Enhancements on the algorithm were proposed by Wriggers and Simo (1990). Another method of interest is the critical displacement method (CDM) which was developed in recent years by Oñate and Matias (1996). Here, the secant stiffness matrix is used to make a prediction of the critical displacements first. Then, in the second step the critical load is computed.

The CDM and the extended system proved to give good results, however both are not perfect and have certain disadvantages. The CDM as an indirect method only predicts the critical values, but gives good results even at greater distances from the critical point. The extended system as direct method computes the critical points directly, but does not converge in all cases and is dependent on the initial values.

The objective of this article is to apply the computation methods for critical points to more complex mechanical problems involving inequality constraints originating from damage and contact. Therefore, the CDM and the extended system as the most promising techniques will be compared and evaluated. One step prediction of the critical load based on the extended system will be developed that enables a better evaluation. A conceivable combination of both
the methods wil be examined. The idea is to use the prediction of a CDM
computation as starting value for the extended system and enhance the
convergence of the latter.
The ay out of the article is as follows: at furst the basic equations in continuum mechanics and finite elements are given. Then a simple bilinear damage and the.bilateral frictionless contact formulation are.presented briefly. Register for free at https. www scipedja com to dpwnfoad the version without the watermark

The numerical examples section is divided into two parts. The first contains the damage examples, where the critical point detection methods are evaluated. In the second part, only the extended system is applied to contact problems.

\section{Basic equations}

\subsection{Continuum mechanics}

With the deformation energy $W$ for hyperelastic material the principle of the minimum of potential energy with respect to the reference configuration can be stated as:

$$
\Pi_{S}=\int_{\mathscr{B}} W \mathrm{~d} V-\int_{\mathscr{B}} \rho_{0} \hat{\mathbf{b}} \cdot \mathbf{u} \mathrm{d} V-\int_{\partial \mathscr{B}_{\sigma}} \hat{\mathbf{t}} \cdot \mathbf{u} \mathrm{d} A \Rightarrow \operatorname{Min} .
$$

Besides the term for the elastic energy, terms considering the energy of applied body forces $\mathbf{b}$ and surface loads $\mathbf{t}$ have been added. The unknown displacement function is denoted by $\mathbf{u}$. Assuming the St Venant constitutive law, the elastic energy is

$$
W=\frac{1}{2}(\mathbf{S E})=\frac{1}{2}(\mathbf{E}:(\mathbf{D E})) \quad \text { with } \mathbf{E}=\frac{1}{2}\left(\mathbf{F}^{\mathrm{T}} \mathbf{F}-\mathbf{I}\right)
$$


Here $\mathbf{S}$ is the second. Piola-Kirchhoff stress tensor, $\mathbf{D}$ is the fourth order constitutive tensor and $\mathbf{E}$ the Green-Lagrange strain tensor with the deformation gradient $\mathbf{F}=(\mathbf{I}+\operatorname{Grad} \mathbf{u})$. Variation of equation (1) then yields

$$
\delta \Pi_{S}=\int_{\mathscr{B}} \mathbf{E}: \mathbf{D} \delta \mathbf{E} \mathrm{d} V-\int_{\mathscr{B}} \rho_{0} \hat{\mathbf{b}} \cdot \delta \mathbf{u} \mathrm{d} V-\int_{\partial \mathscr{B}_{\sigma}} \hat{\mathbf{t}} \cdot \delta \mathbf{u} \mathrm{d} A=0
$$

where the variation of the Green-Lagrange strain tensor is

$$
\delta \mathbf{E}=\frac{1}{2}\left(\mathbf{F}^{\mathrm{T}} \operatorname{Grad} \delta \mathbf{u}+\operatorname{Grad}^{\mathrm{T}} \delta \mathbf{u} \mathbf{F}\right)
$$

and $\delta \mathbf{u}$ is the test function for which $\left\{\delta \mathbf{u} \mid \delta \mathbf{u}=0\right.$ on $\left.\partial \mathscr{B}_{u}\right\}$ is required.

\subsection{FEM}

For the application of the FEM to equation (3) the area of integration $\mathscr{B}$ is approximated by the discretization
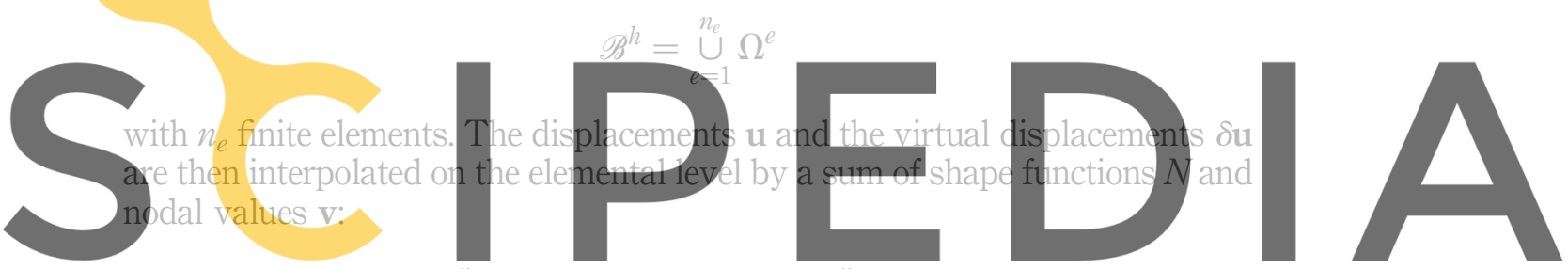

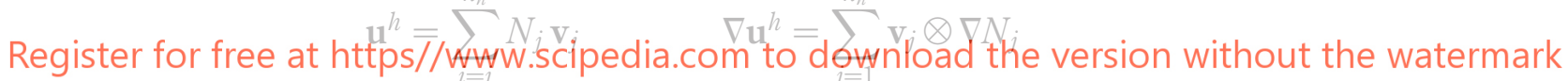

$$
\delta \mathbf{u}^{h}=\sum_{j=1}^{n_{n}} N_{j} \delta \mathbf{v}_{j} \quad \nabla \delta \mathbf{u}^{h}=\sum_{j=1}^{n_{n}} \delta \mathbf{v}_{j} \otimes \nabla N_{j}
$$

with the matrix $\hat{\mathbf{B}}$ that contains derivatives of shape functions according to the theory used, equation (3) can be split into inner energy terms and load terms:

$$
\begin{gathered}
\mathbf{R}(\mathbf{v})=\bigcup_{e=1}^{n_{e}} \int_{\Omega^{e}} \hat{\mathbf{B}}^{\mathrm{T}}\left(\mathbf{v}_{e}\right): \mathbf{S}\left(\mathbf{v}_{e}\right) \mathrm{d} V \\
\mathbf{P}(\mathbf{v})=\bigcup_{e=1}^{n_{e}}\left[\int_{\Omega^{e}} \rho \hat{\mathbf{b}} \mathrm{d} V+\int_{\partial \Omega^{e} \sigma} \hat{\mathbf{t}} \mathrm{d} A\right]
\end{gathered}
$$

This leads finally to the set of equations that has to be solved for the unknown nodal displacements

$$
\mathbf{G}_{S}(\mathbf{v})=\mathbf{R}(\mathbf{v})-\lambda \mathbf{P}=0
$$


EC

$20,5 / 6$

\section{4}

where the load scaling parameter $\lambda$ for equilibrium path computations has already been introduced. Commonly this equation set is solved with the Newton-Raphson method. Due to the complexity of the governing equations the load-deflection path exhibits slopes so that arclength methods for the curve tracing become necessary (Crisfield, 1981; Ramm, 1981; Riks, 1972; Schweizerhof and Wriggers, 1986). In this work, the arclength method with an updated normal plane control equation is applied.

\subsection{Damage model}

In order to compare the critical point detection method for more complex problems a damage model has been implemented. For simplicity reasons the single parameter isotropic damage model of Oliver et al. (1990) is chosen. In combination with the CDM this model was used previously by Tschöpe (2001).

In the constitutive law the scalar damage parameter $d$ is introduced which ranges from 0 (undamaged material) to 1 (completely damaged material):

$$
\mathrm{S}=(1-d) \mathrm{DE}=\overline{\mathrm{DE}}
$$

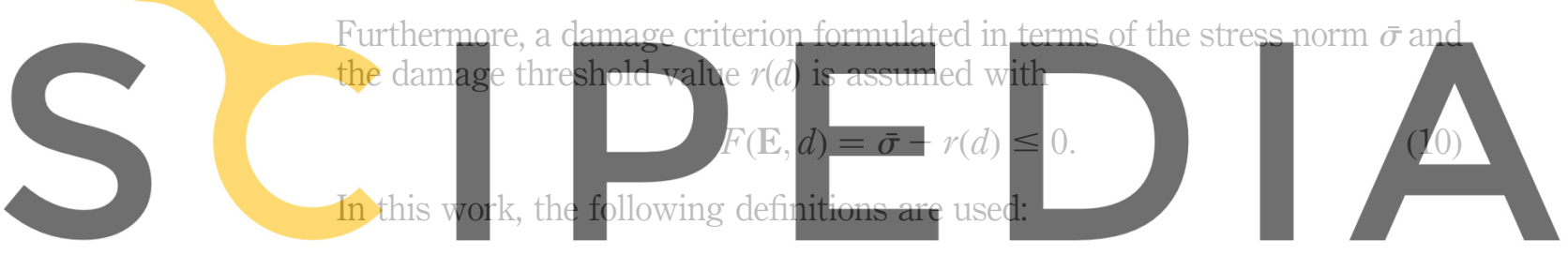

$\bar{\sigma}(\mathrm{E})=\sqrt{\mathbb{E}:(\mathbb{D E})}$

Register for free at https//www.scipedia.com to download the version without the watermark

$$
r(d)=\frac{r_{0}}{1-(1+H) d}
$$

In this equation, $r_{0}$ is the initial damage threshold value and $H$ is the hardening modulus. This damage model is a simple bilinear one where the evolution law for the damage parameter yields

$$
d=\frac{1}{1+H}\left(1-\frac{\tau^{0}}{\bar{\sigma}}\right) .
$$

An algorithm for the application of this damage model can be found in the work of Tschöpe (2001).

\subsection{Contact}

For contact modeling, a two-dimensional frictionless normal contact formulation is used. Therefore, the master-slave concept of Hallquist (1979) is adopted, where one of the bodies is chosen as the master $\mathscr{B}^{\mathrm{M}}$, the other as the slave $\mathscr{B}$. In numerical contact mechanics this is a rather common model, that 
can be found in several articles (Curnier, 1984; Laursen and Simo, 1993; Wriggers and Miehe, 1992).

For a given slave node $\mathbf{x}^{\mathrm{S}}$ on $\partial \mathscr{B}_{c}^{\mathrm{S}}$ a distance function $d\left(\xi_{\alpha}\right)$ to the parameterized master surface $\mathbf{x}^{\mathrm{M}}\left(\xi_{\alpha}\right)$ can be defined. The corresponding master point $\mathbf{x}^{\mathrm{M}}\left(\bar{\xi}_{\alpha}, t\right)$ for the slave point $\mathbf{x}^{\mathrm{S}}$ can be found minimizing this distance function

$$
d\left(\xi_{\alpha}\right)=\left\|\mathbf{x}^{\mathrm{S}}-\mathbf{x}^{\mathrm{M}}\left(\xi_{\alpha}\right)\right\| \Rightarrow \text { Min } \quad \text { with } \alpha=1,2 .
$$

This leads to the local gap function $g_{\mathrm{N}}$, which permits to distinguish between the different contact states:

$$
g_{N}=\left[\mathbf{x}^{\mathrm{S}}-\overline{\mathbf{x}}^{\mathrm{M}}\right] \cdot \overline{\mathbf{n}}
$$

\section{The outward directed normal vector $\overline{\mathbf{n}}$ is obtained from the convective} coordinate system of the surface discretization

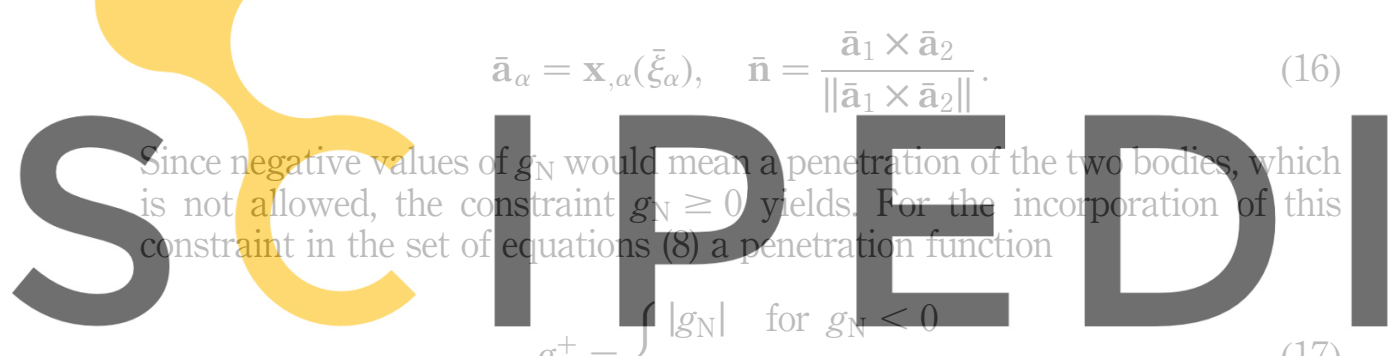

(17)
Direct computation of instability points

615

\section{Register for free at https//www.scipedia.com to download the version without the watermark}

is defined. With this function, the penalty method can be applied and the contact constraints are added to the equation system (8):

$$
G=G_{\mathrm{S}}+\underbrace{\int_{\partial B_{\mathrm{c}}} \varepsilon_{\mathrm{N}} g_{\mathrm{N}}^{+} \delta g_{\mathrm{N}}^{+} \mathrm{d} A}_{\text {contact terms } G_{\mathrm{C}}} \quad \text { with } \varepsilon_{\mathrm{N}}>0
$$

The penalty method does not solve the problem exactly, it only regularizes it. The great advantage of this method is the ease of implementation in the existing finite element program codes. However, due to the regularization tiny rest penetrations remain after the problem is solved. The penalty parameter, $\varepsilon_{\mathrm{N}}$, has to be chosen properly, so that on one side ill conditioning of the equation system and on the other side too large rest penetration do not occur.

For the numerical examples computed in this work a node to segment contact discretization has been used. In general, the description of contact presented can be found in the work of Wriggers (1995) and several other publications. 
EC

$20,5 / 6$

616

\section{Computation of critical points}

This section briefly presents the three critical point computation methods, which will be applied to numerical examples in the end of this work.

\section{$3.1 C D M$}

The CDM was developed recently by Oñate and Matias (1995). Here only an outline of the theory of this method is given. For a more detailed description, the reader may refer to Oñate and Matias (1996) and Matias (1996).

The CDM is based on the secant stiffness matrix, which is derived by introducing the displacements of an updated configuration as $\mathbf{u}^{t+\Delta t}=$ $\mathbf{u}^{t}+\Delta \mathbf{u}$. With these displacements an incremental Green-Lagrange strain tensor yields:

$$
\Delta \mathbf{E}=\mathbb{E}^{t+\Delta t}-\mathbf{E}^{t}=\xi+\eta
$$

In the tensors, $\xi$ and $\eta$, the terms with linear and quadratic dependence on $\Delta \mathbf{u}$ are summarized, so that

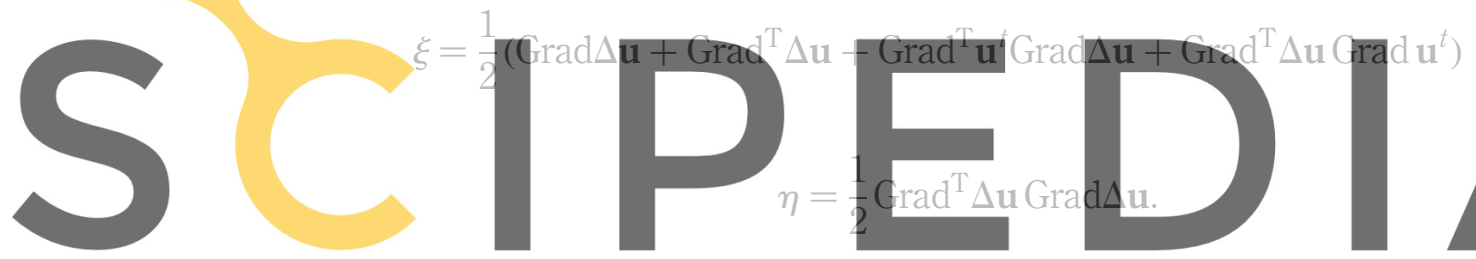

Deriving a similar expression for $\delta \mathbf{E}$ and doing the incremental split for the

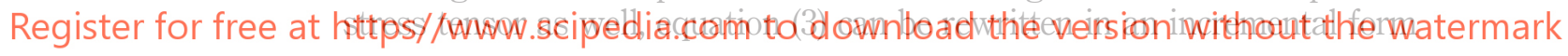

$$
\begin{aligned}
& \int_{B_{0}}\left[\delta \xi^{\mathrm{T}} \mathbf{D} \xi+\delta \xi^{\mathrm{T}} \mathbf{D} \boldsymbol{\eta}+\delta \boldsymbol{\eta}^{\mathrm{T}} \mathbf{D} \xi+\delta \eta^{\mathrm{T}} \mathbf{D} \boldsymbol{\eta}+\delta \eta^{\mathrm{T}} \mathrm{S}^{t}\right] \mathrm{d} V \\
& \quad=\int_{B_{0}} \delta \Delta \mathbf{u}^{\mathrm{T}} \hat{\mathbf{b}}^{t+\Delta t} \rho_{0} \mathrm{~d} V+\int_{\partial B_{0} \sigma} \delta \Delta \mathbf{u}^{\mathrm{T}} \hat{\mathbf{T}}^{t+\Delta t} \mathrm{~d} A-\int_{B_{0}} \delta \xi^{\mathrm{T}} \mathbf{S}^{t} \mathrm{~d} V .
\end{aligned}
$$

Similar to the interpolation in equation (5) the incremental displacement are approximated by

$$
\Delta \mathbf{u}^{h}=\sum_{i=1}^{n_{n}} N_{i} \Delta \mathbf{v}_{i}, \quad \delta \Delta \mathbf{u}^{h}=\sum_{i=1}^{n_{n}} N_{i} \delta \Delta \mathbf{v}_{i} .
$$

After some mathematical operations (Oñate, 1995; Oñate and Matias, 1995) the secant stiffness matrix can be assembled as the sum of the following four matrices:

$$
\mathbf{K}_{\mathrm{S}}=\mathbf{K}_{\mathrm{LU}}+\mathbf{K}_{\mathrm{M}}+\mathbf{K}_{\mathrm{N}}+\mathbf{K}_{\sigma}
$$


Details of the form of the above matrices can be found in the work of Oñate and Matias (1996).

As expected the limiting behavior yields the tangent stiffness matrix

$$
\mathbf{K}_{\mathrm{T}}=\lim _{\Delta \mathbf{v} \rightarrow 0} \mathbf{K}_{\mathrm{S}}=\mathbf{K}_{\mathrm{LU}}+\mathbf{K}_{\sigma} .
$$

To obtain a prediction of the critical values it is assumed that the critical displacements are equal to

$$
\mathbf{v}_{c}=\mathbf{v}^{t}+\Delta \mathbf{v} \quad \text { with } \Delta \mathbf{v}=\rho \Phi .
$$

The secant stiffness matrix is now assembled with this relation. Neglecting the terms with quadratic and higher dependence on $\rho$ the eigenvalue problem $\mathrm{K}_{\mathrm{T}} \Phi=0$ can be approximated with this secant matrix by

$$
\left[\mathbf{K}_{\mathrm{T}}\left(\mathbf{v}^{t}\right)+\rho\left(\mathbf{K}_{\mathrm{L} 2}+\mathbf{K}_{\sigma 1}\right)\right] \Psi=0 .
$$

The form of all the above matrices can be found in the work of Matias (1996) and Oñate and Matias (1996).

Solving this eigenvalue problem with the inverse iteration method a
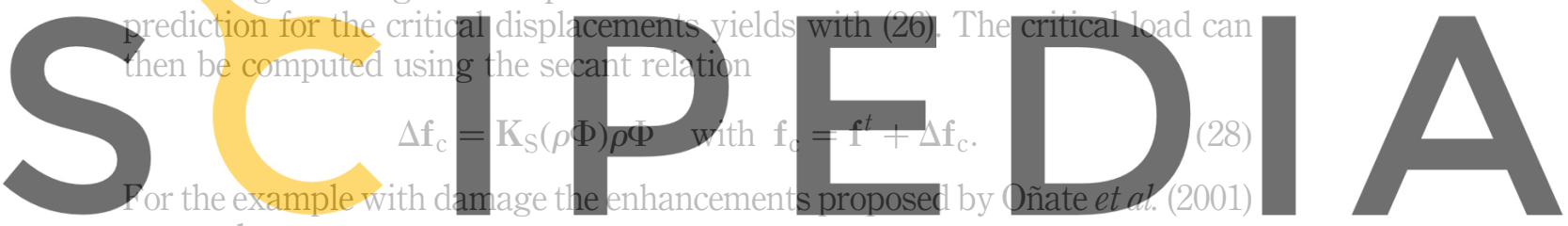

are used:

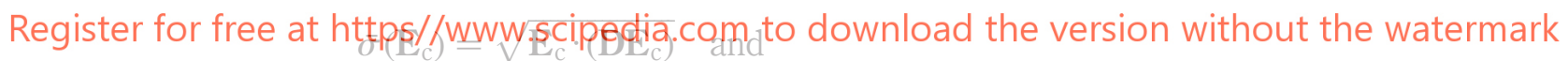

$$
d_{\mathrm{c}}= \begin{cases}a^{t} & \text { for } \bar{\sigma}\left(\mathbf{E}_{\mathrm{c}}\right) \leq r^{t} \\ \frac{1}{1+H}\left(1-\frac{r_{0}}{\bar{\sigma}\left(\mathbf{E}_{\mathrm{c}}\right)}\right) & \text { for } \bar{\sigma}\left(\mathbf{E}_{\mathrm{c}}\right)>r^{t}\end{cases}
$$

The basic idea with this is to compute equation (28) based on the damage state of the predicted critical displacements.

\subsection{Extended system}

The application of the extended system to damage problems is straightforward in contrast to contact problems where the additional terms in equation (8) pose some difficulties. The solution of equation systems of the type (8) and (18) is accomplished with the Newton-Raphson method. This standard finite element algorithm for equation (18) reads as

$$
\left[\mathbf{K}_{\mathrm{T}}\left(\mathbf{v}^{i}\right)+\mathbf{K}_{\mathrm{c}}\left(\mathbf{v}^{i}\right)\right] \Delta \mathbf{v}^{i+1}=-\mathbf{G}_{\mathrm{S}}\left(\mathbf{v}^{i}\right)-\mathbf{G}_{\mathbf{c}}\left(\mathbf{v}^{i}\right)
$$

with 
EC

$20,5 / 6$

$$
\mathbf{v}^{i+1}=\mathbf{v}^{i}+\Delta \mathbf{v}^{i+1}
$$

The matrices $\mathbf{K}_{\mathrm{T}}$ and $\mathbf{K}_{\mathrm{c}}$ are the contributions to the global tangent stiffness matrix originating from the solid and contact terms $\mathbf{G}_{\mathrm{S}}$ and $\mathbf{G}_{c}$, respectively:

\section{8}

$$
\mathbf{K}_{\mathrm{T}}=\frac{\partial \mathbf{G}_{\mathrm{S}}}{\partial \mathbf{v}}, \quad \mathbf{K}_{\mathrm{c}}=\frac{\partial \mathbf{G}_{\mathrm{c}}}{\partial \mathbf{v}}
$$

For the direct computation of instability points with the extended system, the equation system (18) is extended by a condition for the critical point. In this work, the eigenvector equation in the critical point $\mathbf{K}_{\mathrm{T}} \phi=0$ was chosen. This procedure has been described before in literature in several publications, (Wriggers and Smio, 1990; Wriggers and Wagner, 1989; Wriggers et al., 1988). With the additional constraint and a scaling equation for the eigenvector, the extended equation system is
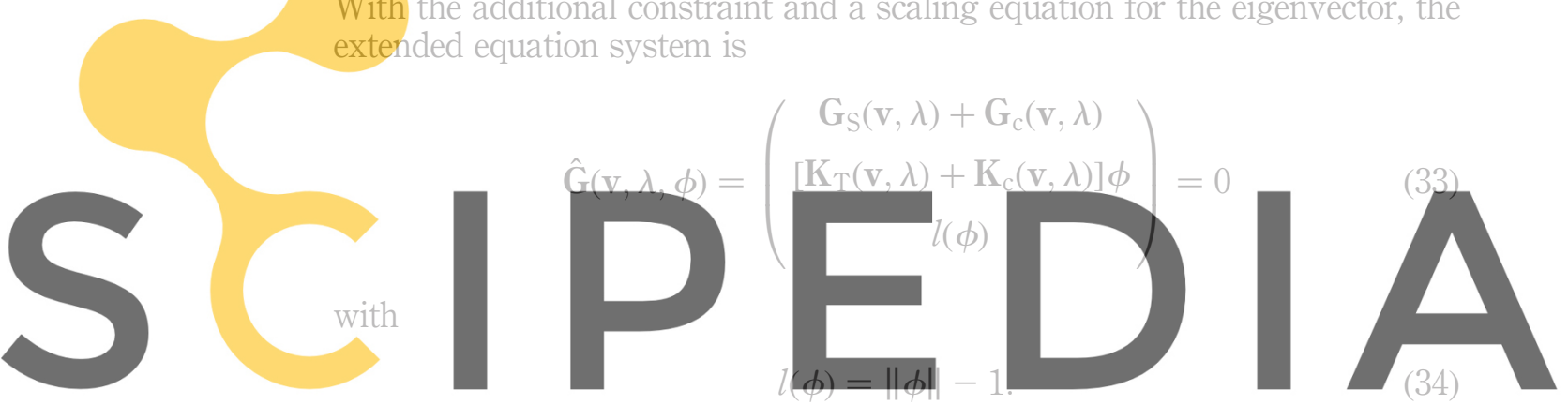

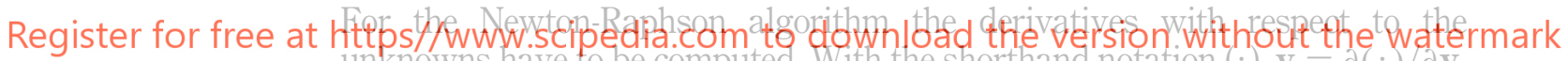
unknowns have to be computed. With the shorthand notation ('), v $=\partial(\cdot) / \partial \mathrm{v}$, equation (30) evolves to:

$$
\begin{aligned}
& {\left[\begin{array}{ccc}
\mathbf{K}_{\mathrm{T}}+\mathbf{K}_{\mathrm{c}} & 0 & -\mathbf{P} \\
{\left[\left(\mathbf{K}_{\mathrm{T}}+\mathbf{K}_{\mathrm{c}}\right) \phi\right], \mathbf{v}} & \mathbf{K}_{\mathrm{T}}+\mathbf{K}_{\mathrm{c}} & {\left[\left(\mathbf{K}_{\mathrm{T}}+\mathbf{K}_{\mathrm{c}}\right) \phi\right], \lambda} \\
0^{\mathrm{T}} & \frac{\phi^{\mathrm{T}}}{\|\phi\|} & 0
\end{array}\right]\left(\begin{array}{c}
\Delta \mathbf{v} \\
\Delta \phi \\
\Delta \lambda
\end{array}\right)} \\
& =-\left(\begin{array}{c}
\mathbf{G}_{\mathrm{S}}(\mathbf{v})+\mathbf{G}_{\mathrm{c}}(\mathbf{v}) \\
{\left[\mathbf{K}_{\mathrm{T}}(\mathbf{v})+\mathbf{K}_{\mathrm{c}}(\mathbf{v})\right] \phi} \\
\|\phi\|-1
\end{array}\right)
\end{aligned}
$$

The number of unknowns now has increased to $2 n+1$ with $n$ being the degrees of freedom of the equation system (18). For the efficient solution of (35) (Wriggers and Simo, 1990; Wriggers et al., 1988) proposed an algorithm with a numerical derivative as an approximation for the derivatives of $\mathbf{K}_{\mathrm{T}}$. 
This algorithm is adapted to the contact problems of this paper and outlined in the Appendix.

In this algorithm, special attention is necessary for the reassemblation of the stiffness matrix $\mathbf{K}_{\mathrm{c}}\left(\mathbf{v}^{i}+\varepsilon \phi^{i}\right)$ in combination with the contact model used in this work. Since for each assembly of the stiffness matrix a new mapping of slave nodes to master segments is performed in the contact algorithm, this means that $\mathbf{K}_{\mathrm{c}}\left(\mathbf{v}^{i}\right)$ and $\mathbf{K}_{\mathrm{c}}\left(\mathbf{v}^{i}+\varepsilon \phi^{i}\right)$ can differ substantially. To prevent this the assembly of $\mathbf{K}_{\mathrm{c}}\left(\mathbf{v}^{i}+\varepsilon \phi^{i}\right)$ is made based on the master segment to slave node mapping of $\mathbf{K}_{\mathrm{c}}\left(\mathbf{v}^{i}\right)$. Furthermore, the active set of $\mathbf{K}_{c}\left(\mathbf{v}^{i}\right)$ is held fix, too. The negative effect of adhesive forces that are applied for a degree of freedom in the active set whose gap was closed in $\mathbb{K}_{c}\left(\mathbb{v}^{i}\right)$ but become open in $\mathbb{K}_{c}\left(\mathbf{v}^{i}+\varepsilon \phi^{i}\right)$ are negligible due to the low magnitude of $\varepsilon \phi$. A proper choice of the starting values for the extended system is of great importance for the convergence of the algorithm. For the eigenvector $\phi$ several possible starting vectors exist:

P

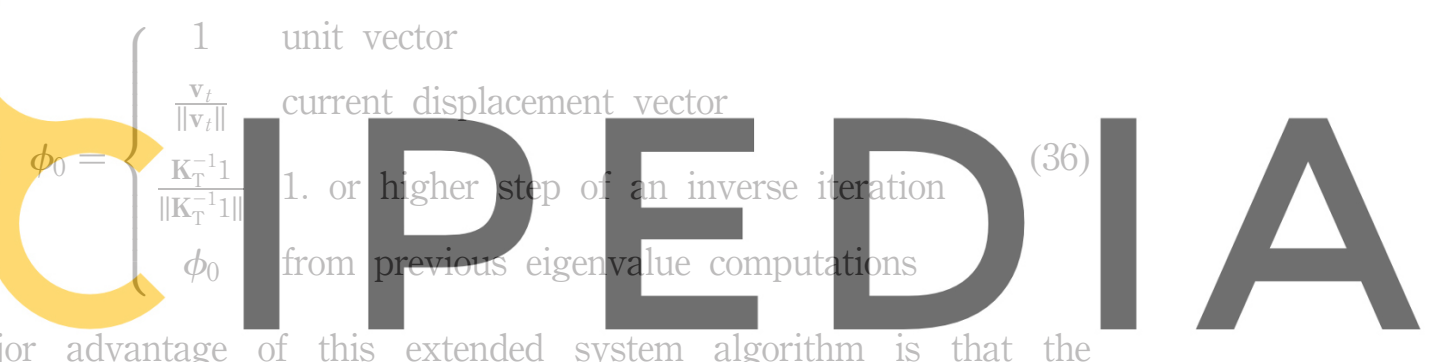

A major advantage of this extended system algorithm is that the computation of instability points

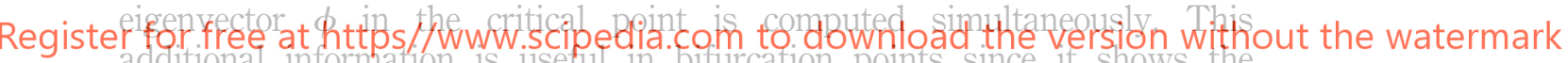
deformation pattern of the secondary path. This deformation pattern is needed for path switching algorithms.

\subsection{One step prediction}

The one step prediction consists basically of the first step of the application of the extended system algorithm. Rewriting equation system (35) for the first iteration and setting $\hat{\mathbf{K}}_{\mathrm{T}}=\mathbf{K}_{\mathrm{T}}+\mathbf{K}_{\mathrm{c}}$ for the following set of three equations can be obtained:

$$
\begin{gathered}
\hat{\mathbf{K}}_{\mathrm{T}}\left(\mathbf{v}^{0}\right) \Delta \mathbf{v}^{1}=\Delta \lambda^{1} \mathbf{P} \\
{\left[\hat{\mathbf{K}}_{\mathrm{T}}\left(\mathbf{v}^{0}\right) \phi\right], \mathbf{v} \Delta \mathbf{v}^{1}+\hat{\mathbf{K}}_{\mathrm{T}}\left(\mathbf{v}^{0}\right) \Delta \phi^{1}=-\hat{\mathbf{K}}_{\mathrm{T}}\left(\mathbf{v}^{0}\right) \phi} \\
\phi^{\mathrm{T}} \Delta \phi^{1}=\|\phi\|-\|\phi\|^{2}
\end{gathered}
$$

In these equations, the fact was exploited that $-\mathbf{R}\left(\mathbf{v}^{0}\right)+\lambda^{0} \mathbf{P}-\mathbf{G}_{\mathrm{c}}\left(\mathbf{v}^{0}\right)=0$ when the extended system computation is started from a converged 
EC

$20,5 / 6$

620

$$
\Delta \lambda^{1}=-\frac{1}{\frac{\phi^{\mathrm{T}}}{\|\phi\|} \hat{\mathbf{K}}_{\mathrm{T}}^{-1}\left[\left(\hat{\mathbf{K}}_{\mathrm{T}} \phi\right), \mathbf{v} \Delta \mathbf{v}_{\mathrm{P}}^{1}\right]} \quad \text { so that } \lambda^{1}=\lambda^{0}+\Delta \lambda^{1} .
$$

This means that an estimation of the critical load $\lambda^{1}$ can be obtained this way. The computation of the denominator in equation (40) can be simplified again by the numerical derivative as in the extended system algorithm (Appendix). The eigenvector $\phi$ in equation (40) is one of the possible starting vectors from equation (36).

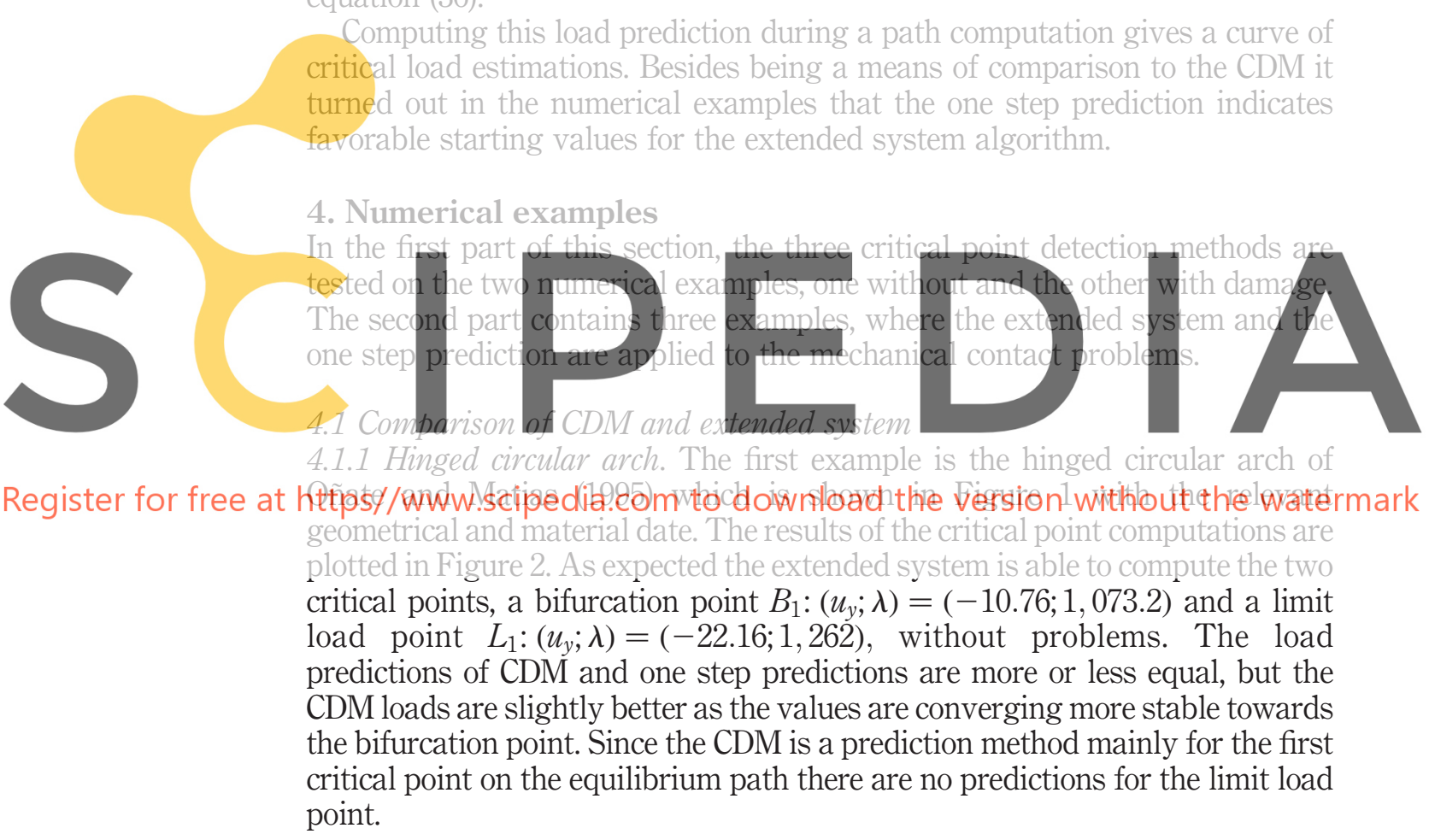

Figure 1.

Hinged circular arch with solid elements

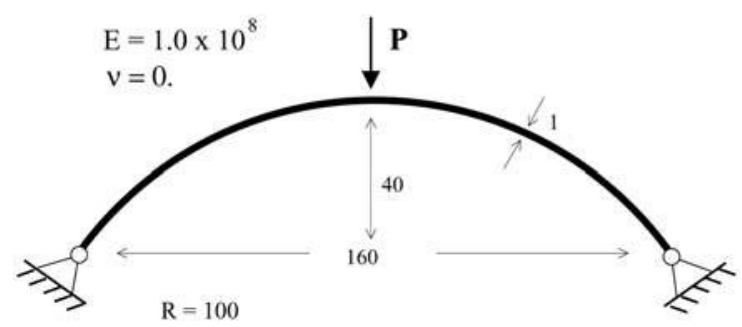




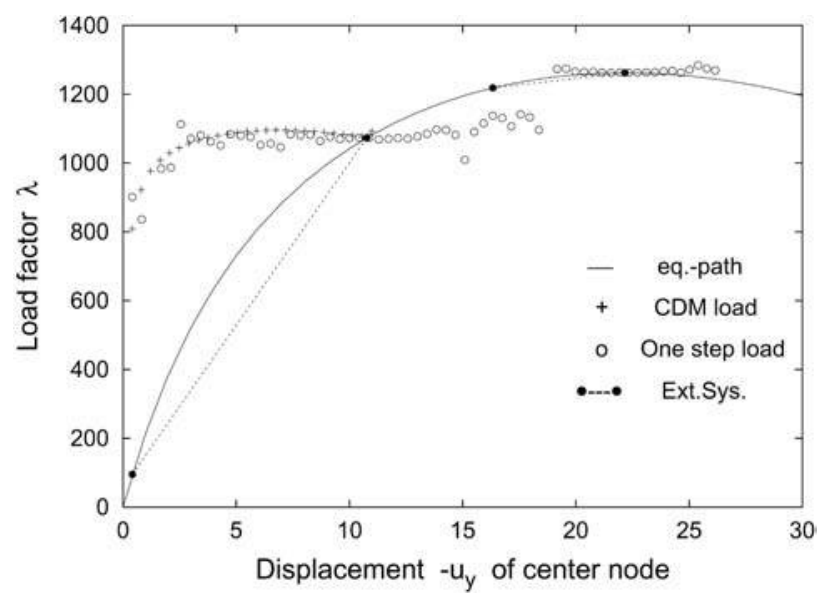

\section{Direct computation of instability points}

621

Figure 2. Equilibrium path for the hinged circular arch

4.1.2 Clamped shallow arch. As a second example, the clamped shallow arch from Oñate et al. (2001) is chosen. An outline including geometrical and material data is given in Figure 3. A hardening parameter of $H=1.5$ and an initial threshold value of $r_{0}=0.2$ are assumed.

The computational results are shown in Figure 4. With the extended system the limit load point at $L_{1}:\left(u_{y} ; \lambda\right)=(-8.56 ; 110.29)$ is obtained easily. As in the previous example, the $\mathrm{CDM}$ critical load predictions are slightly better than the one step prediction loads. The enhancements of the critical load prediction with damage (mentioned at the end of Section 3.1) become noticeable in this example, since the CDM values are lesser than the one step prediction values.

\subsection{Examples with contact}

The two examples of the previous section showed that the CDM loads and the one step prediction loads were more or less equal with a slight advantage for the $\mathrm{CDM}$ values. A disadvantage of the $\mathrm{CDM}$ is the huge effort for the implementation of the CDM in the existing finite element codes where the secant stiffness matrix commonly is not assembled. Considering this,

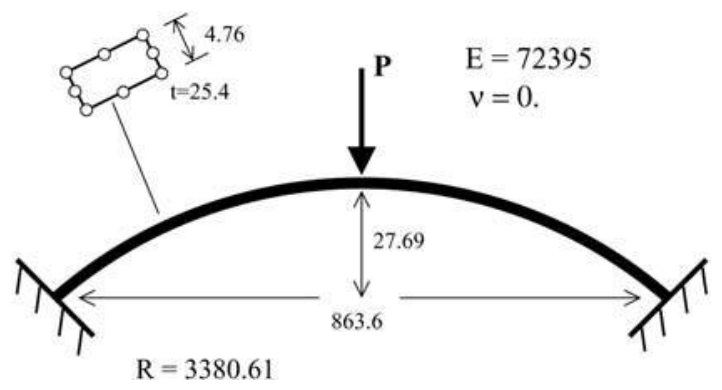

Figure 3.

Clamped shallow arch with solid elements 
EC

$20,5 / 6$

622

Figure 4.

Equilibrium path of the shallow arch with

damage $r_{0}=0.2, H=1.5$

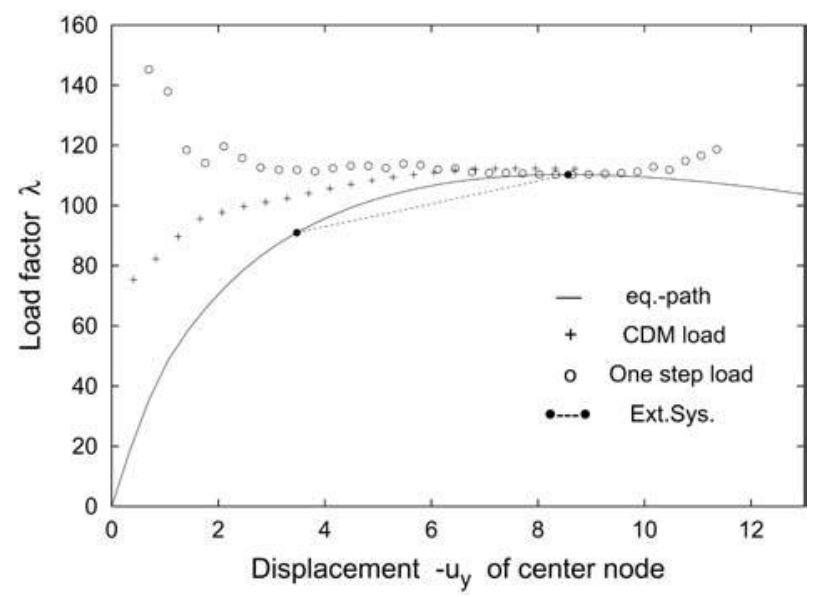

the slightly better results do not justify the effort necessary for the CDM. Therefore, only the extended system is used for the examples of this section.

4.2.1 Hinged arch with obstacles. The first example with contact is an arch with two obstacles placed above and below it at one side. Figure 5 shows an outline of the structure including geometrical and material data. A load is placed in the apex and the distance between obstacles and arch is 0.1 . The arch is discretized with 20 layers of 300 Q1-elements each, the obstacles consist of 100 elements in ten layers.

Looking at the results in Figure 6 the common bifurcation point for hinged arches is not present due to the obstacles and only a limit load point at $\left(-u_{y} ; \lambda\right)=(-6.97 ; 245.0)$ remains. The extended system is able to compute this point directly from some distance. The one step prediction is quite good. After some initial up and down the curve stabilizes for points with $-u_{y}>3.5$. This means that favorable starting values for the extended system are in the range $3.5<-u_{y}<8$. This results in Figure 6 confirm this.

Figure 5.

Hinged arch with obstacles

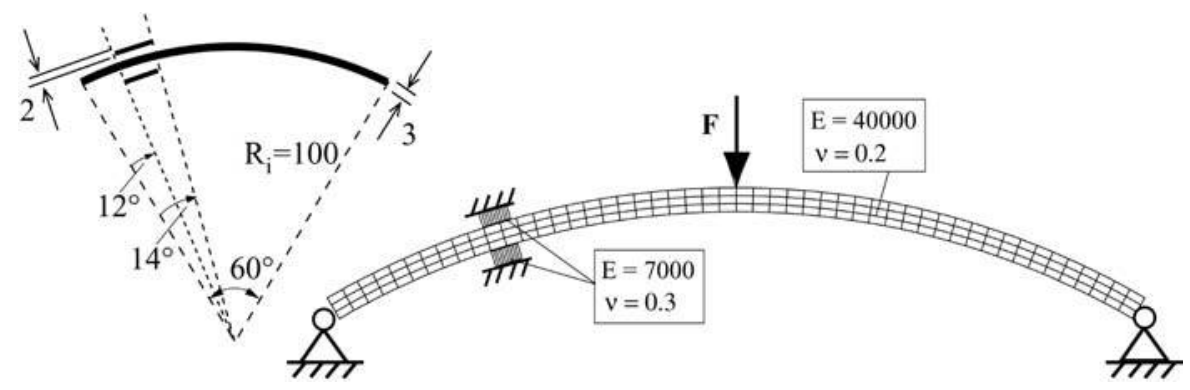




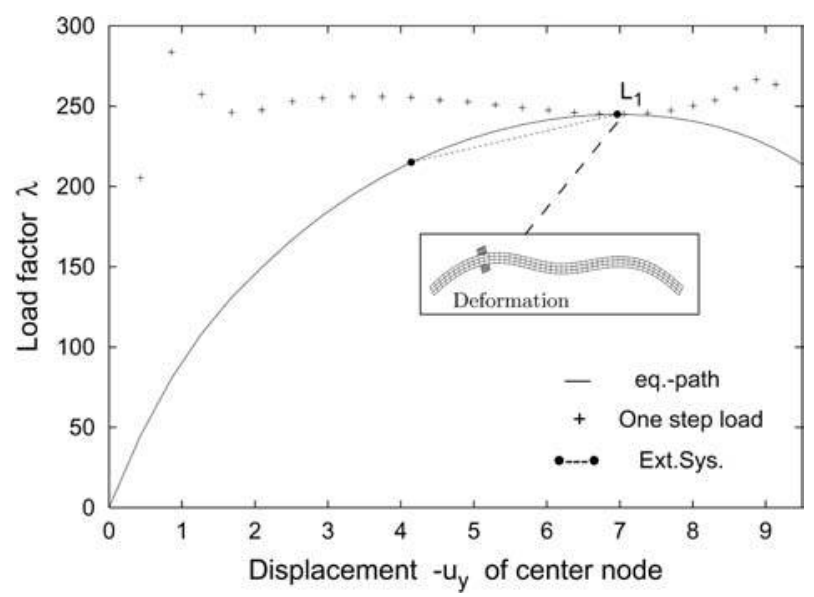

\section{Direct computation of instability points}

623

Figure 6.

Equilibrium path for the hinged arch

4.2.2 Two arches. The next example is the structure of an arch that has a second arch upside down on top of it. An outline containing geometrical and material data is shown in Figure 7. A sidewards movement ( $x$-direction) of the upper arch is prevented by the boundary conditions in the corner nodes, where the unit loads are applied.

The arches were discretized with four node elements with linear shape functions. Covergence studies of this example proved that an elementation with 14,000 elements is sufficient for the exactness of the numerical solution.

The results of the extended system computations are shown in Figure 8. Two bifurcation points with $B_{1}:\left(u_{y} ; \lambda\right)=(-0.85 ; 17.21)$ and $B_{2}:\left(u_{y} ; \lambda\right)=$ $(-4.77 ; 75.94)$ and two limit load points with $L_{1}:\left(u_{y} ; \lambda\right)=(-12.94 ; 108.83)$ and $L_{2}:\left(u_{y} ; \lambda\right)=(-47.73 ; 42.14)$ were found successfully. The dashed lines in Figure 8 symbolize the application of the extended system. Starting point and converged end point are marked with dots.

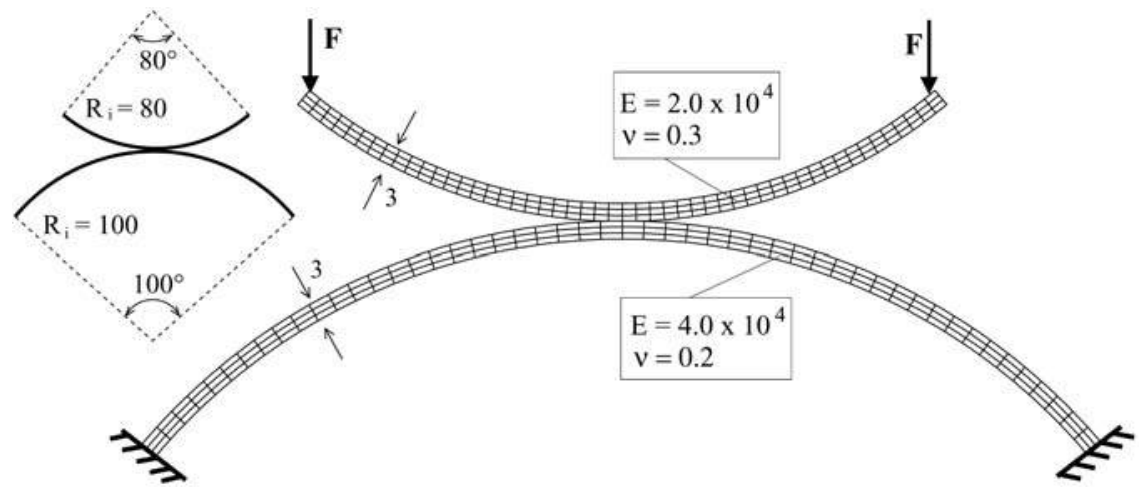

Figure 7.

Outline of the two arches 
EC

$20,5 / 6$

624

Figure 8.

Equilibrium path with critical points

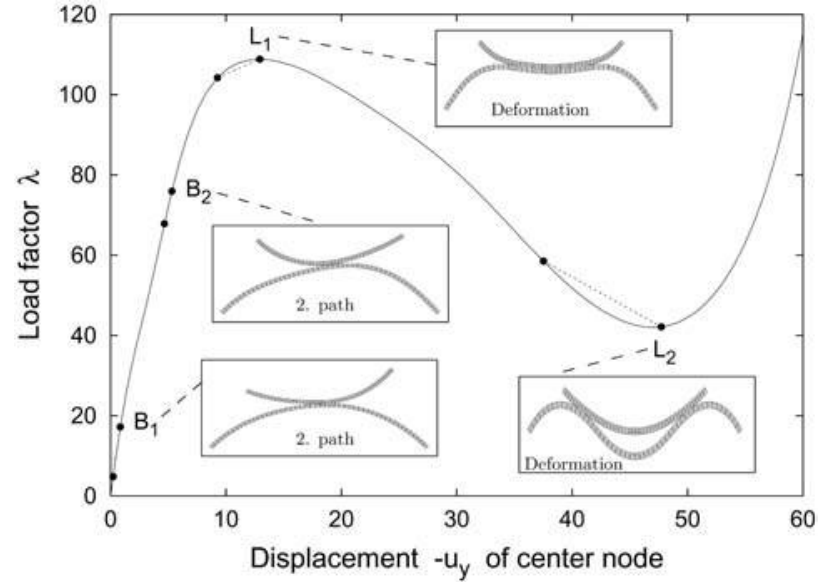

The deformed arches and the secondary path deformation, respectively, are depicted in the small pictures next to each critical point. The secondary path deformations are plots of the eigenvectors that are among the results of the extended system computation.

In Figure 9 the curves of the critical loads predictions calculated with the one step prediction algorithm are plotted. Comparing these with Figure 8 it can be seen how the load predictions indicate for each starting point to which critical point the extended system algorithm converges.

4.2.3 Deep arch with obstacles. The geometrical data of the hinged deep arch with two arches as obstacles are outlined in Figure 10. The obstacles are

Figure 9.

One step prediction for the two arches structure

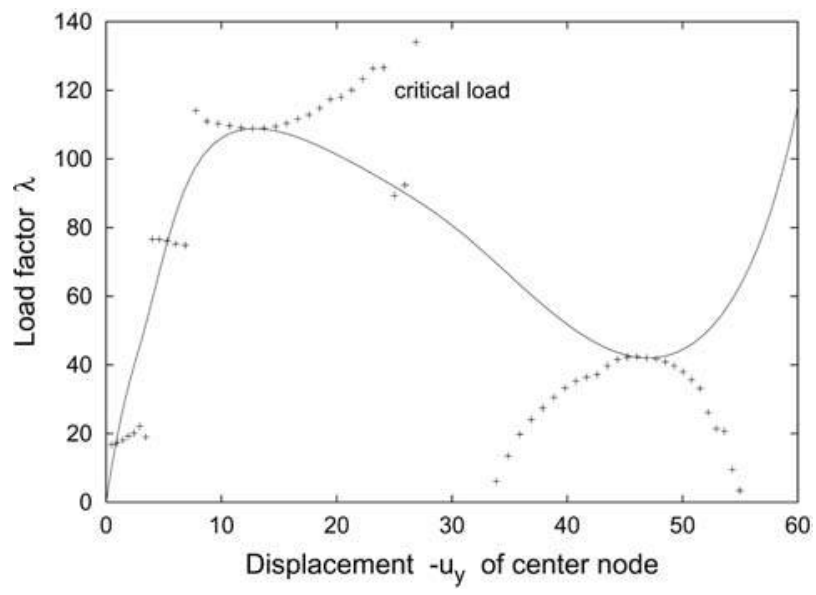




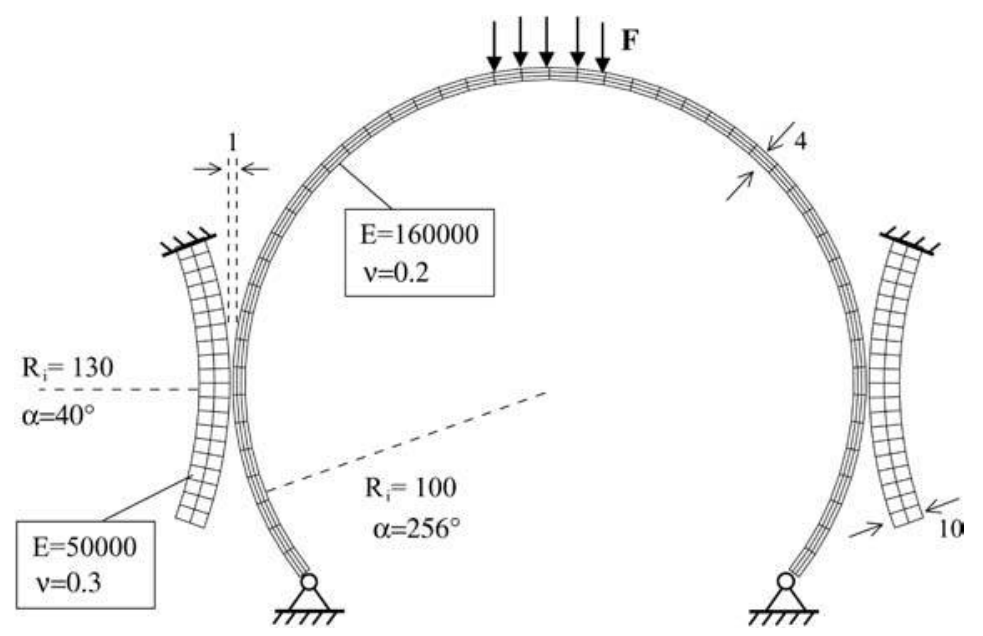

\section{Direct computation of instability points}

625

Figure 10.

Outline of the deep arch with obstacles

clamped on one side. The elementation of the deep arch is 12 layers with 600 elements each, the obstacles have 300 elements in eight layers. Here also unit loads are placed in the 41 center nodes of the deep arch.

The two limit load points $L_{1}:\left(u_{y} ; \lambda\right)=(-50.44 ; 20.94)$ and $L_{2}:\left(u_{y} ; \lambda\right)=$ $(-144.4 ; 8.74)$ can be found with the extended system, as demonstrated in Figure 11. The extended system computations for the points are started from a not to far distance as indicated by the one step predictions.

\section{Conclusions}

In the article, the computation of critical points for inequality constrained problems was presented. The inequality constraints were imposed by

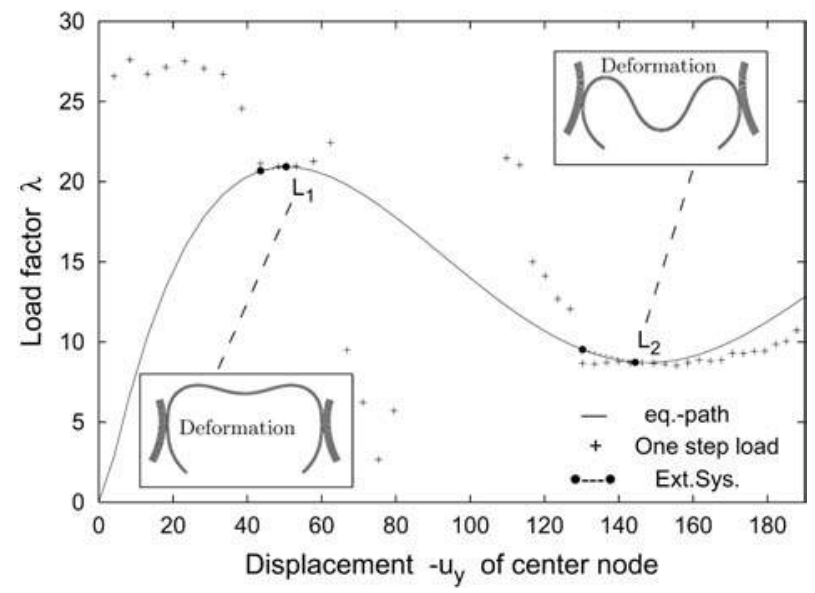

Figure 11. Equilibrium path for the deep arch with obstacles 
EC $20,5 / 6$

626

the occurrence of damage and contact. In the first part the CDM and the extended system were evaluated for damage problems with the objective of using the CDM predicted load as an input for the extended system and to enhance the convergence of the latter. A one step prediction method based on the extended system was presented to enable a better comparison of CDM load and common starting values of the extended system. The CDM load was slightly better than the load of the one step prediction method. Weighting this with the high effort for the implementation of the CDM the conclusion of this comparison was that a combination does not offer any substantial advantages. Thus for the contact examples of the second part only the extended system was used. In all examples this method proved capable of computing the critical points directly. Besides this the prediction method developed in the first part served as an indication method for favorable starting values for the extended system.

\section{References}

Abbott, J. (1978), "An efficient algorithm for the determination of certain bifurcation points", Journal of Computational and Applied Mathematics, Vol. 4 No. 1, pp. 19-27.

Crisfield, M. (1981), "A fast incremental/iterative solution procedure that handles snap through", Computers and Structures, Vol. 13, pp. 55-62.

Curnier, A. (1984), "A theory of friction”, International Journal of Solids and Structures, Vol. 20, pp. 637-47.

Euler, L. (1774), Methodus inveniendi lineas curvas maximi minimive propietate gaudentes (Appendix, De curvis elasticis). Lausanne and Geneva.

Hallquist, J. (1979), "Nike2d: an implicit finite deformation, finite element code for analyzing the static and dynamic response on two-dimensional solids", Report UCRL-52678, UCLawrence Livermore National Laboratory.

Laursen, T. and Simo, J. (1993), "A continuum-based finite element formulation for the implicit solution of multibody large deformation fricitional contact problems", International Journal for Numerical Methods in Engineering, Vol. 36, pp. 3451-85.

Matias, W. (1996), "El método de desplazamiento crítico en el análisis de inestabilidad estrucural" $\mathrm{PhD}$ thesis, Departament de resisténcia de materials i estructures a l'enginyeria, Universitat Pollitècnica de Catalunya, Barcelona, Spain.

Oliver, J., Cervera, M., Oller, S. and Lubliner, J. (1990), "Isotropic damage models and smeared crack analysis of concrete", in Nenad Bicanic, et al. (Ed.), Proceedings SCI-C Computer Aided Analysis and Design of Concrete Structures, Pineridge Press, Swansea, pp. 945-58.

Oñate, E. (1995), "On the derivation and possibilities of the secant stiffness matrix for non-linear finite element analysis", Comput. Mechanics, Vol. 15, pp. 572-93.

Oñate, E. and Matias, W. (1995), "Enhanced prediction of structural instability points using a critical displacement method", in Wiberg, N-E. (Ed.), Advances in Finite Element Technology, CIMNE, Barcelona, pp. 62-89.

Oñate, E. and Matias, W. (1996), "A critical displacement approach for predicting structural instability", Computer Methods in Applied Mechanics and Engineering, Vol. 134, pp. 135-61.

Oñate, E., Tschöpe, H. and Wriggers, P. (2001), "Combination of the critical displacement method with a damage model for structural instability analysis", Engineering Computations, Vol. 18 Nos 3/4, pp. 642-62. 
Ramm, E. (1981), "Strategies for tracing the nonlinear response near limit points", in Wunderlich, W., Stein, E. and Bathe, K-J. (Eds), Nonlinear Finite Element Analysis in Structural Mechanics, Springer Verlag, Berlin, Heidelberg, New York, pp. 63-89.

Riks, E. (1972), "The application of newtons method to the problem of elastic stability", Journal of Applied Mechanics, Vol. 39, pp. 1060-6.

Schweizerhof, K. and Wriggers, P. (1986), "Consistent linearization for path following methods in nonlinear FE analysis", Computational Method in Applied Mechanical Engineering, Vol. 59, pp. 261-79.

Seydel, R. (1979), "Numerical computation of branch points in nonlinear equations", Numerische Mathematik, Vol. 33, pp. 339-52.

Tschöpe, H. (2001), "Direct computation of instability points with inequality constraints using the finite element method," $\mathrm{PhD}$ thesis, Institut für Baumechanik und numerische Mechanik, Universität Hannover.

Wemper, G. (1971), "Discrete approximation related to nonlinear theories of solids", International Journal of Solids and Structures, Vol. 7, pp. 1581-99.

Wriggers, P. (1995), Finite Element Algorithms for Contact Problems, Vol. 2, CIMNE, Barcelona, pp. 1-49.

Wriggers, P. and Miehe, C. (1992), "Recent advances in the simulation of thermomechanical contact processes", in Owen, D.R.J., Hinton, E. and Oñate, E. (Eds), Proceedings of COMPLAS III, Pineridge Press, Swansea.

Wriggers, P. and Simo, J. (1990), "A general procedure for the direct computation of turning and bifurcation points”, International Journal for Numerical Methods in Engineering, Vol. 30, pp. 155-76.

Wriggers, P. and Wagner, W. (1989), "Ein quadratisch konvergentes Verfahren zur Berechnung von Stabilitätspunkten", ZAMM, Zeitschrift für angewandte Mathematik und Mechanik, Vol. 69 No. 4, pp. T 219-22.

Wriggers, P., Wagner, W. and Miehe, C. (1988), "A quadratically convergent procedure for the calculation of stability points in finite element analysis", Computer Methods in Applied Mechanics and Engineering, Vol. 70, pp. 329-47.

\section{Appendix. Algorithm for the extended system}

Solve for $\Delta \mathbf{v}^{i+1}$ :

$$
\begin{aligned}
& {\left[\mathbf{K}_{\mathrm{T}}\left(\mathbf{v}^{i}\right)+\mathbf{K}_{\mathrm{c}}\left(\mathbf{v}^{i}\right)\right] \Delta \mathbf{v}_{\mathrm{P}}^{i+1}=\mathbf{P}} \\
& {\left[\mathbf{K}_{\mathrm{T}}\left(\mathbf{v}^{i}\right)+\mathbf{K}_{\mathrm{c}}\left(\mathbf{v}^{i}\right)\right] \Delta \mathbf{v}_{G}^{i+1}=-\mathbf{G}_{\mathrm{S}}\left(\mathbf{v}^{i}\right)-\mathbf{G}_{\mathrm{c}}\left(\mathbf{v}^{i}\right)}
\end{aligned}
$$

Compute:

$$
\begin{aligned}
& \mathbf{h}_{1}^{i}=\frac{1}{\varepsilon}\left[\mathbf{K}_{\mathrm{T}}\left(\mathbf{v}^{i}+\varepsilon \boldsymbol{\phi}^{i}\right) \Delta \mathbf{v}_{\mathrm{P}}^{i+1}+\mathbf{K}_{\mathrm{c}}\left(\mathbf{v}^{i}+\varepsilon \boldsymbol{\phi}^{i}\right) \Delta \mathbf{v}_{\mathrm{P}}^{i+1}-\mathbf{P}\right] \\
& \mathbf{h}_{2}^{i}=\frac{1}{\varepsilon}\left[\mathbf{K}_{\mathrm{T}}\left(\mathbf{v}^{i}+\varepsilon \boldsymbol{\phi}^{i}\right) \Delta \mathbf{v}_{\mathrm{G}}^{i+1}+\mathbf{K}_{\mathrm{c}}\left(\mathbf{v}^{i}+\varepsilon \boldsymbol{\phi}^{i}\right) \Delta \mathbf{v}_{\mathrm{G}}^{i+1}+\mathbf{G}_{\mathrm{S}}\left(\mathbf{v}^{i}\right)+\mathbf{G}_{\mathrm{c}}\left(\mathbf{v}^{i}\right)\right]
\end{aligned}
$$

Solve for $\Delta \phi^{i+1}$ : 

EC
$20,5 / 6$
$\left[\mathbf{K}_{\mathrm{T}}\left(\mathbf{v}^{i}\right)+\mathbf{K}_{\mathrm{c}}\left(\mathbf{v}^{i}\right)\right] \Delta \phi_{1}^{i+1}=-\mathbf{h}_{1}^{i}$
$\left[\mathbf{K}_{\mathrm{T}}\left(\mathbf{v}^{i}\right)+\mathbf{K}_{\mathrm{c}}\left(\mathbf{v}^{i}\right)\right] \Delta \phi_{2}^{i+1}=-\mathbf{h}_{2}^{i}$

Compute increments:

\section{8}

$$
\begin{aligned}
& \Delta \lambda^{i+1}=\frac{-\phi^{i^{\mathrm{T}}} \Delta \phi_{2}^{i+1}+\left\|\phi^{i}\right\|}{\phi^{i^{\mathrm{T}}} \Delta \phi_{1}^{i+1}} \\
& \Delta \mathbf{v}^{i+1}=\Delta \lambda^{i+1} \Delta \mathbf{v}_{\mathrm{P}}^{i+1}+\Delta \mathbf{v}_{\mathrm{G}}^{i+1}
\end{aligned}
$$

Update:

$$
\begin{aligned}
& \lambda^{i+1}=\lambda^{i}+\Delta \lambda^{i+1}, \quad \mathbf{v}^{i+1}=\mathbf{v}^{i}+\Delta \mathbf{v}^{i+1}, \\
& \phi^{i+1}=\Delta \lambda^{i} \Delta \phi_{1}^{i+1}+\Delta \phi_{2}^{i+1}
\end{aligned}
$$


This article has been cited by:

1. Konstantinos Tzaros, Euripidis Mistakidis. 2015. The constrained buckling problem of geometrically imperfect beams: a mathematical approach for the determination of the critical instability points. Meccanica 50:5, 1263-1284. [CrossRef]

2. Ricardo A.M. Silveira, Wellington L.A. Pereira, Paulo B. Gonçalves. 2008. Nonlinear analysis of structural elements under unilateral contact constraints by a Ritz type approach. International Journal of Solids and Structures 45:9, 2629-2650. [CrossRef] 